\title{
Choroidal Metastasis Secondary to Testicular Mature Teratoma: A Case Report
}

\author{
Bashar M. Bata Sachin M. Salvi \\ Sheffield Ocular Oncology Service, Department of Ophthalmology, Sheffield Teaching Hospitals, Sheffield, UK
}

\section{Established Facts}

- Testicular germ cell tumours (TGCTs) are the most common solid malignancies in young males.

- Choroidal metastasis secondary to TGCTs is extremely rare and often are associated with haemorrhage and highly elevated.

- Bleomycin, etoposide, and cisplatin (BEP) chemotherapy with adjuvant radiotherapy is effective in treating choroidal metastasis secondary to TGCTs.

\section{Novel Insights}

- A rare case of choroidal metastasis secondary to mature teratoma, a subtype of TGCTs.

- Systemic symptoms and signs aid in reaching a correct diagnosis when TGCTs first present with visual symptoms.

- Restoration of normal visual acuity and normal macular anatomy can be achieved using BEP without the need for adjuvant radiotherapy.

\section{Keywords}

Testicular germ cell tumours · Choroidal metastasis ·

Mature teratoma

\section{Abstract}

We report a case of a choroidal metastasis secondary to testicular mature teratoma, a subtype of non-seminoma germ cell tumours, in a young adult male. The choroidal metastasis was treated successfully with bleomycin, etoposide, and cisplatin chemotherapy without the need for adjuvant radiotherapy. This represents a rare case of mature teratoma metastasizing to the choroid and one of very few reported testicular germ cell tumours to achieve normal or near normal visual acuity with chemotherapy alone despite severe macular disease at presentation.

(c) 2021 S. Karger AG, Basel

\section{Introduction}

Testicular germ cell tumours (TGCTs) are the most common solid malignancies in young males. Uveal metastasis secondary to testicular tumours is extremely rare with only 24 reported cases since 1936 . The vast majority of these cases were choriocarcinomas in patients known to have a testicular tumour, who either died soon after diagnosis or did not achieve good visual acuity after treatment. We present an unusual case of mature teratoma presenting with visual symptoms secondary to uveal metastasis in a young male, whose visual acuity was restored to $6 / 6$ after treatment with only chemotherapy. 


\section{Case Report}

A 28-year-old man was referred to the ocular oncology clinic with a 4-week history of painless progressive loss of vision in the right eye. He also complained of recent diffuse skin rash, bilateral breast lumps, and constant headaches. He had no previous ophthalmic or medical history and no family history of cancer but had 2 pack-year history of smoking.

On examination, he had visual acuity of 3/60 in the right eye and $6 / 4$ in the left. Fundus examination revealed an amelanotic creamy-coloured choroidal mass lesion that involved the macula and extended temporally towards the equator of the right eye with overlying subretinal fluid but no lipofuscin pigment or surface drusen (Fig. 1a). There were no similar lesions in the other eye. Bscan ultrasonography revealed a dome-shaped choroidal lesion with a basal diameter of $14 \times 14 \mathrm{~mm}$ and a height of $5 \mathrm{~mm}$ but no evidence of choroidal excavation (Fig. 2c). The lesion had variable internal reflectivity on A-scan ultrasonography (Fig. 2a) and intraretinal fluid overlying it on ocular coherence tomography (OCT) (Fig. 2b).

Examination of the skin revealed diffuse, confluent, erythematous, and scaly skin rash (Fig. 1b) while examination of the chest revealed bilateral gynaecomastia. An ultrasound scan of the testicles showed an ill-defined, irregular, heterogenous mass within the left testicle suggestive of primary neoplasia of the testicle (Fig. 2f). Computed tomography (CT) scan of the thorax, abdomen, and pelvis revealed multiple pulmonary metastases (Fig. 2e) and an enlarged cystic lymph node in the paracaval area. MRI of the brain uncovered 4 mass lesions, 1 right occipital, 2 left occipital, and 1 right frontal, suggestive of cerebral metastasis (Fig. 2d).
The serum human chorionic gonadotropin (beta-hCG) level was significantly elevated $(29,977 \mathrm{IU} / \mathrm{L})$ whereas lactate dehydrogenase (LDH) was only slightly raised (541 IU/L). A diagnosis of non-seminomatous germ cell tumour (NSGCT) of the testicle, with metastases to the lungs, brain, and choroid (stage III), was made on the basis of the blood markers and the radiographic findings.

Five days after presenting to the eye clinic, he underwent stereotactic radiosurgery to his brain lesions and received the first of 4 cycles of bleomycin, etoposide, and platinum (BEP) chemotherapy. Following an initial response to chemotherapy, his tumour markers plateaued; hence, he was switched to ifosfamide, carboplatin, and etoposide (ICE). However, ICE chemotherapy was suspended after he developed a large macular haemorrhage in his left eye secondary to chemotherapy-induced thrombocytopenia. $\mathrm{He}$ then underwent left orchiectomy after a whole-body positron emission tomography scan with the glucose analogue 2-fluoro2-deoxy-D-glucose (FDG-PET) confirmed no metabolic activity in all 3 sites of metastasis. Histopathology showed post-chemotherapy mature teratoma with no evidence of viable or semi-viable germ cell tumour.

His visual acuity in the right eye improved rapidly after starting chemotherapy and reached 6/9 after only 2 cycles. At his most recent follow-up visit to the eye clinic, 1 month following orchiectomy and 9 months after initiation of chemotherapy, he had no ocular symptoms. His visual acuity was $6 / 5$ in both eyes. Fundus examination of both eyes was completely normal except for an area of chorioretinal atrophy temporal to the macula in the right eye (Fig. 3). He remains under surveillance to monitor him for recurrence of the treated metastatic lesions.
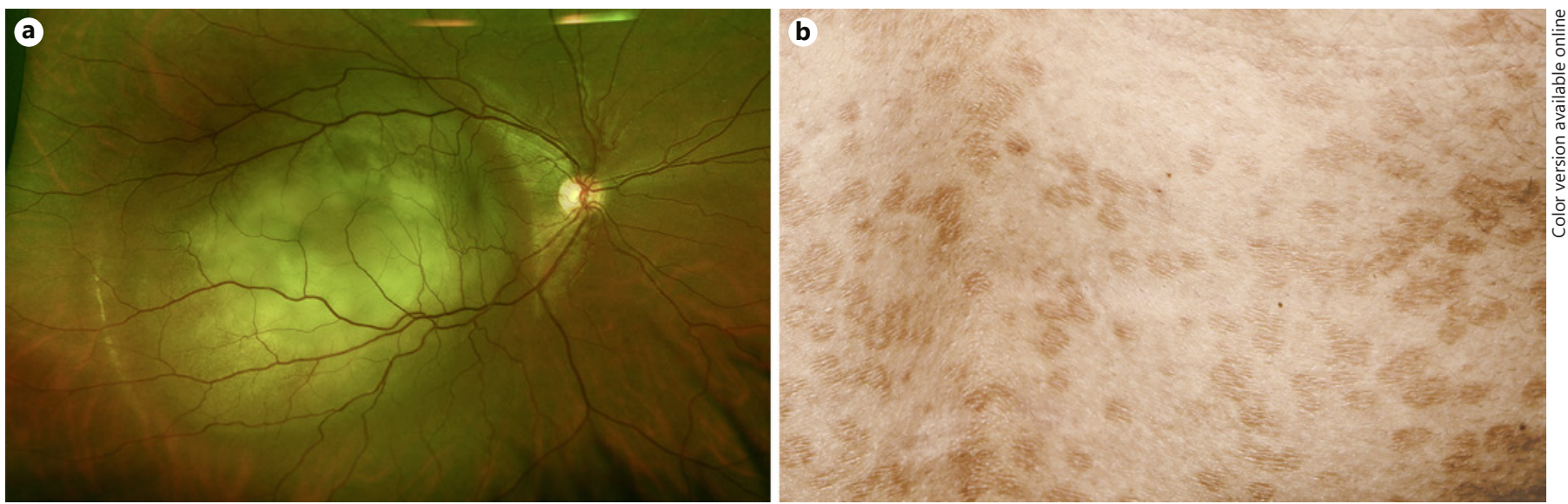

Fig. 1. At presentation (a) wide field fundus photo of the right eye showed posterior pole creamy-coloured choroidal lesion. $\mathbf{b}$ Diffuse macular skin rash.

Fig. 2. a An A-scan showed variable internal reflectivity. b EDIOCT showed a choroidal lesion with overlying intraretinal fluid. c A longitudinal B-scan ultrasonography at 9 o'clock showed a heterogenous choroidal mass lesion without choroidal excavation. d A T2-weighted MRI of the brain showed right frontal and right occipital metastatic lesions. e A CT scan of the chest showed bilateral pulmonary metastatic lesions. f An ill-defined, irregular, heterogenous mass within the left testicle that was $1.46 \times 1.01 \mathrm{~cm}$ in dimensions. EDI-OCT, extended depth ocular coherence tomography; CT, computed tomography.
(For figure see next page.) 

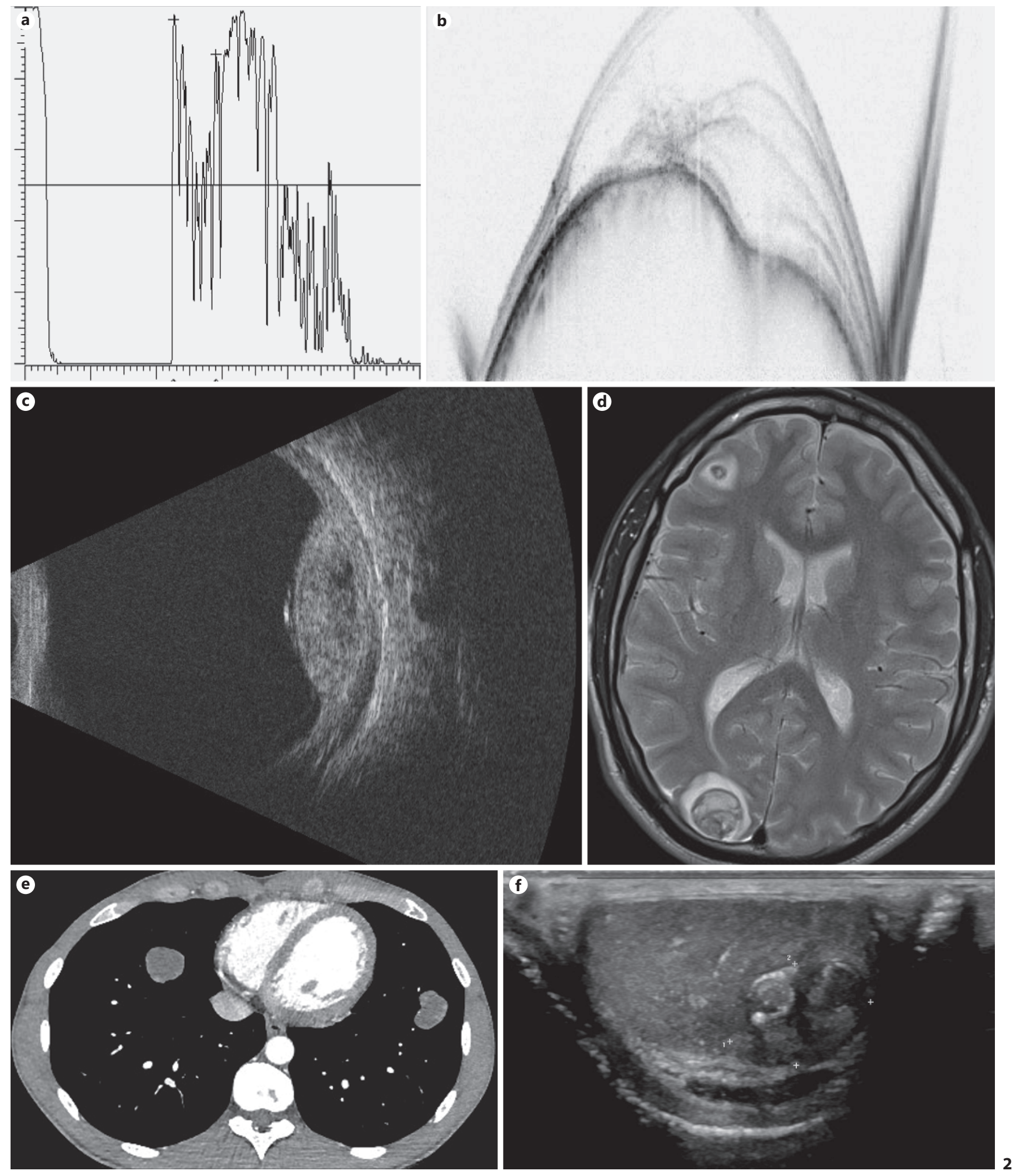

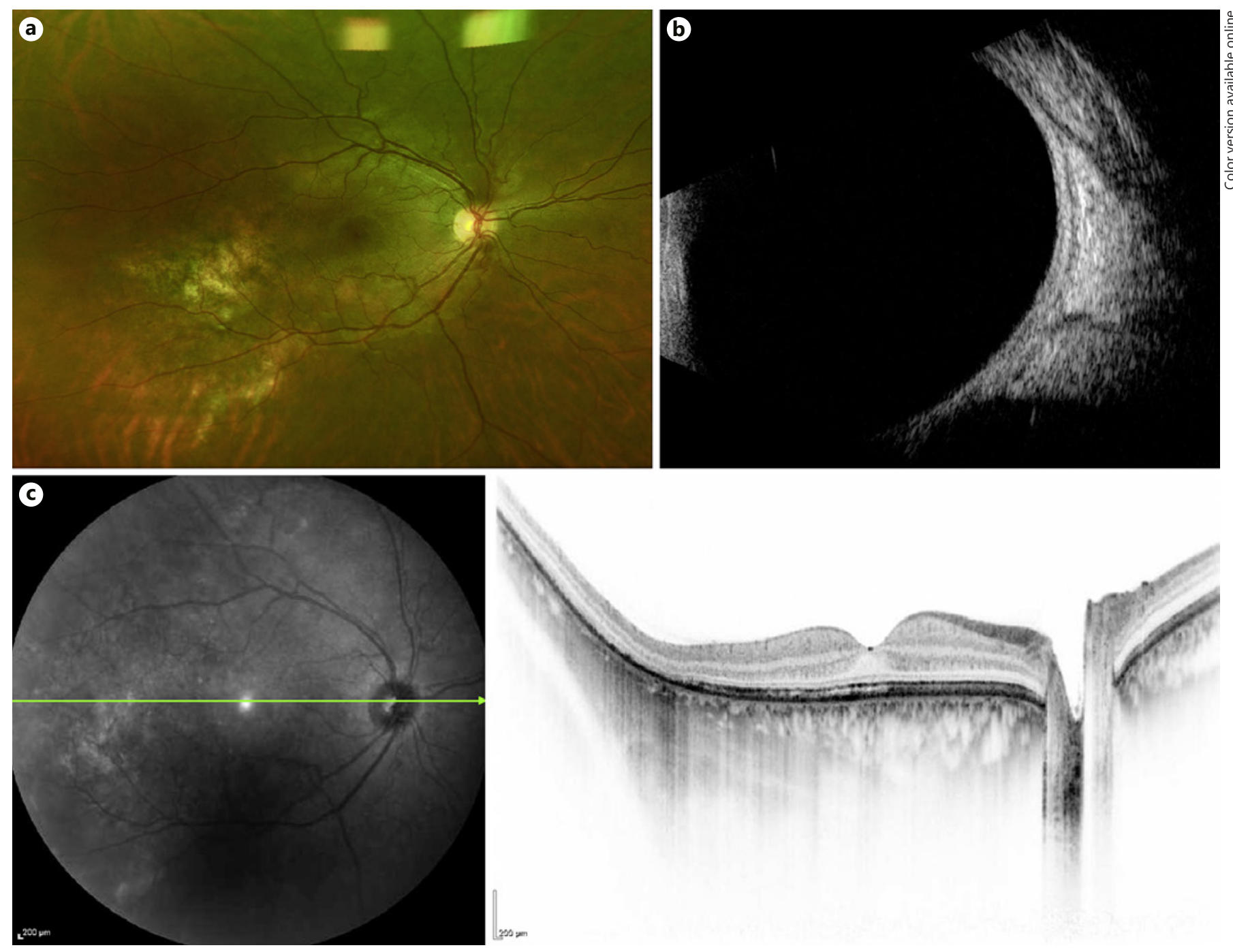

Fig. 3. Nine months after presentation (a) wide field fundus photo of the right eye showed resolution of the macular metastatic lesion with adjacent area of chorioretinal atrophy. b A longitudinal B-scan ultrasonography at 9 o'clock showed the resolution of the choroidal lesion. c EDI-OCT at the macula of the right eye showed normal foveal anatomy. EDI-OCT, extended depth ocular coherence tomography.

\section{Discussion}

TGCT, which comprise $98 \%$ of all testicular tumours, are the most common solid tumours in adolescent and young adult males [1]. Their incidence has increased in the recent years, particularly among men of European ancestry $[2,3]$. At initial diagnosis, approximately two thirds of patients have stage I disease, which means no lymph node involvement, no distant metastasis, and normal levels of tumour markers. One-third of patients are diagnosed with metastatic disease. The most common sites for metastasis are lungs, distant lymph nodes, liver, brain, and bone [4]. Patients with non-pulmonary visceral me- tastases are classed as poor risk and have an overall 5-year survival of $48 \%$ [5].

Uveal metastasis secondary to testicular tumours, first reported by Macdonald in 1936 [6], is rare. A meta-analysis published in 2006 listed 16 cases reported between the years 1936 and 2005 [7]. Search of PubMed and Ovid using different combinations of the terms uveal metastasis, choroidal metastasis, testicular cancer, germ cell tumour, teratoma, choriocarcinoma, and chorioepithelioma revealed that 8 more cases have been reported since [8-14]. Most previously reported cases were choriocarcinomas, a subtype of NSGCT. Our case represents a rare case of uveal metastasis secondary to mature teratoma, a 
different subtype of NSGCT. Mature teratomas are more common in prepubertal children and are usually benign. However, when present in adults they are at risk of metastasis.

Despite its rarity, TGCT should be suspected when a young male patient presents with choroidal metastasis. The majority of reported cases were unilateral but bilateral disease has been reported as well $[11,12,15]$. These lesions were noted to be haemorrhagic [7] and highly elevated $[16,17]$. Both features were absent in our patient, hence cannot be relied upon to make a diagnosis. Instead, early identification of systemic symptoms and signs, such as gynaecomastia and skin rash, was the key in reaching the correct diagnosis quickly and promptly administrating chemotherapy that allowed him to achieve complete remission. Gynaecomastia in patients with TGCT results from high beta-HCG level, whereas the skin rash is likely paraneoplastic since it appeared briefly before ocular symptoms and resolved with treatment. However, it could have also been an incidental finding and unrelated to the testicular tumour.

External beam radiotherapy (EBRT) has historically been the most frequently used treatment modality for uveal metastasis. However, it carries a risk of cataract, radiation retinopathy, exposure keratopathy, and optic neuropathy. Several studies have shown that chemotherapy alone may lead to partial or complete regression of choroidal metastasis $[18,19]$. Zech and colleagues were the first to report success in treating choroidal metastasis secondary to TGCT using BEP chemotherapy without adjuvant radiotherapy [16]. Their patient, however, ended up with 20/1,000 vision due to a chorioretinal scar. Three subsequent case reports showed that chemotherapy alone is capable of achieving good visual outcome [1012]. Similarly, our patient showed rapid response to chemotherapy as his vision improved to near normal after only his second cycle. By the time he completed the fourth cycle he did not have any ocular symptoms, his visual acuity was restored to and maintained at $6 / 6$, and the foveal normal anatomy was restored. This provides further evidence that chemotherapy alone can be enough to treat choroidal metastasis secondary to TGCT. Given our patient had a solitary lesion, he could have had stereotactic radiosurgery at the same setting in which his brain metastasis was treated, but this would have meant loss of vision in that eye. Thus, we suggest that radiotherapy is reserved for patients who show no or incomplete response to chemotherapy.

In conclusion, TGCT should be suspected when a young male presents with uveal metastasis. Prompt diagnosis and administration of chemotherapy is essential to achieve remission and good visual outcome.

\section{Statement of Ethics}

The subject gave his written consent to publish his case (including publication of images).

\section{Conflict of Interest Statement}

The authors have no conflicts of interest to declare.

\section{Funding Sources}

The authors did not receive any funding.

\section{Author Contributions}

Bashar M. Bata contributed to the idea, collected the data, prepared the figures, and wrote the manuscript. Sachin Salvi contributed to the idea and to writing the manuscript.

\section{References}

1 Fitzmaurice C, Fitzmaurice C, Allen C, Barber RM, Barregard L, Bhutta ZA, et al. Global, regional, and national cancer incidence, mortality, years of life lost, years lived with disability, and disability-adjusted life-years for 32 cancer groups, 1990 to 2015: a systematic analysis for the global burden of disease study. JAMA Oncol. 2017;3(4):524-48.

2 Siegel RL, Miller KD, Jemal A. Cancer statistics, 2016. CA Cancer J Clin. 2016;66:7-30.
3 Ghazarian AA, Trabert B, Devesa SS, McGlynn KA. Recent trends in the incidence of testicular germ cell tumors in the united states. Andrology. 2015;3(1):13-8.

$4 \mathrm{Xu} \mathrm{P}$, Wang J, Abudurexiti $\mathrm{M}$, Jin S, Wu J, Shen Y, et al. Prognosis of patients with testicular carcinoma is dependent on metastatic site. Front Oncol. 2019;9:1495.

5 International germ cell consensus classification: a prognostic factor-based staging system for metastatic germ cell cancers. International germ cell cancer collaborative group. J Clin Oncol 1997;15(2):594-603.
6 Macdonald AE. Choroidal chorionepithelioma secondary to teratoma of the testicle. Trans Am Ophthalmol Soc. 1936;34:11721.

7 Kavanagh MC, Pakala SR, Hollander DA, O'Brien JM. Choriocarcinoma metastatic to the choroid. Br J Ophthalmol. 2006;90(5): 650-2.

8 Piccinin MR, Almeida JA Jr, Aydos RD, Nogueira DC, Silva RF. [choroid metastasis of testicular primary site: case report]. Arq Bras Oftalmol. 2006;69(6):949-53.
Choroidal Metastasis Secondary to

Testicular Mature Teratoma
Ocul Oncol Pathol 2021;7:85-90 DOI: $10.1159 / 000511597$ 
9 Purandare NC, Sanghvi DA, Thakur MH. Choroidal metastasis from non-seminomatous germ cell tumour of the testis. Br J Radiol. 2008;81(967):e188-90.

10 Khurana RN, Dibernardo C, Handa JT. Improved systemic chemotherapy for metastatic testicular choriocarcinoma can result in excellent prognosis for life and vision. Arch Ophthalmol. 2008;126(7):1008-9.

11 Senapati SN, Samanta DR, Das PK, Byondyopadhyay A, Mohantya A. Choroid and cutaneous metastasis from a testicular tumor. $\mathrm{He}$ matol Oncol Stem Cell Ther. 2008;1(4):24951.
12 Guber I, Zografos L, Schalenbourg A. Choroidal metastases in testicular choriocarcinoma, successful treatment with chemo- and radiotherapy: a case report. BMC Urol. 2011;11:24.

13 Bains S, Jain A, Sharma K. Choroidal metastasis as the presenting feature in a case of testicular choriocarcinoma. Saudi J Ophthalmol. 2012;26(2):249-51.

14 Barba-Navarrete DM, Moreno-Páramo D, Corona-Montes VE, Tapia-López LE, Montiel-Delgado E. Ophthalmological changes of the posterior segment in patients with testicular cancer in a mixed-race population of the general hospital of mexico. Arch Soc Esp Oftalmol. 2018;93(9):417-22.

15 Chitwood EM Jr. Chorioepithelioma of testis with bilateral choroidal metastasis. AMA Arch Ophthalmol. 1953;50(3):363-7.
16 Zech JC, Subiger L, Chiquet C, Bouvier R, Trepsat C. Testicular choriocarcinoma metastatic to the choroid. Retina. 1999;19(2):164-

17 Osada K, Iijima H, Imasawa M, Takahashi $\mathrm{H}$ Kobori Y, Nakagomi H, et al. Metastatic uveal tumor secondary to testicular choriocarcinoma. Jpn J Ophthalmol. 2004;48(1):85-7.

18 Letson AD, Davidorf FH, Bruce RA Jr. Chemotherapy for treatment of choroidal metastases from breast carcinoma. Am J Ophthalmol. 1982;93(1):102-6.

19 Manquez ME, Brown MM, Shields CL, Shields JA. Management of choroidal metastases from breast carcinomas using aromatase inhibitors. Curr Opin Ophthalmol. 2006; 17(3):251-6. 